\title{
Effect of modulation-doping on luminescence properties of plasma assisted MBE-grown GaN/AIGaN quantum well
}

\author{
N. Kamata ${ }^{1, a}$, H. Klausing ${ }^{2}$, F. Fedler ${ }^{2}$, D. Mistele ${ }^{2}$, J. Aderhold ${ }^{2}$, O. K. Semchinova ${ }^{2}$, J. Graul ${ }^{2}$, \\ T. Someya ${ }^{3}$, and Y. Arakawa ${ }^{3}$ \\ 1 Dept. of Functional Materials Science, Saitama University, Saitama-shi, Saitama 338-8570, Japan \\ ${ }^{2}$ Lab. für Informationstechnologie, Universität Hannover, Schneiderberg, Hannover 30167, Germany \\ 3 Research Center for Advanced Science and Techn., Univ. of Tokyo, Meguro-ku, Tokyo 153-8904, Japan
}

Received: 4 July 2003 / Accepted: 28 January 2004 - C EDP Sciences

\begin{abstract}
In order to improve the crystal quality of GaN-based light emitting devices, photoluminescence (PL) characterization of below-gap states in plasma assisted MBE-grown GaN/AlGaN quantum well (QW) structures has been done by utilizing a below-gap excitation (BGE) light in addition to an above-gap excitation light. The decrease of the band-edge PL intensity due to the addition of the BGE of $1.17 \mathrm{eV}$ indicates the presence of an energy-matched below-gap state in the two-wavelength excited PL. In continuation to our previous efficiency improvement by applying modulation-doping to GaAs/AlGaAs QW's, we focused on several undoped and Si-doped GaN/AlGaN QW's. Experimental results showed that $\mathrm{Si}$ modulation-doping reduces the density of below-gap states in the QW region, hence it is promising for increasing internal quantum efficiency of GaN-based QW's.
\end{abstract}

PACS. 78.55.Cr III-V semiconductors - 78.67.De Quantum wells - 71.55.Eq III-V semiconductors

\section{Introduction}

In spite of a considerable strain due to lattice-mismatch between substrate and a difficulty of controlling residual imperfections, continuing developments of crystal growth technology have brought us remarkable progress in InGaN/GaN and GaN/AlGaN light emitting devices for the green, blue and ultraviolet wavelength region $[1,2]$. Various types of buffer-layers and lateral growth schemes are found effective for reducing densities of dislocations and defects in the upper active region. Silicon doping is important not simply for controlling majority carrier concentration, but for relaxing internal stress and reducing imperfections during growth in GaN-based materials [2].

In order to utilize wide band-gap and direct-transition properties, it is still crucial to understand the mechanism of grown-in defects and residual imperfections which are detected electronically and optically as below-gap states. Further improvement of emission efficiency and reliability is expected if below-gap states acting as nonradiative recombination (NRR) centers or trap centers can be eliminated during crystal growth and device processing. Here modulation-doping of Si into GaN/AlGaN QW was shown to be promising for reducing defect density based on a two-wavelength excited photoluminescence (PL) measurement.

\section{Principles of two-wavelength excited photoluminescence}

In two-wavelength excited PL (TWEPL), two light sources with photon energies higher and lower than the band-gap of the sample were provided for an above-gap excitation (AGE) and a below-gap excitation (BGE), respectively. The BGE light changes the electronic population of an energy-matched below-gap state in the sample, which shifts the NRR rate via the below-gap state and hence modulates the band-edge PL intensity originating from the AGE light (i.e. BGE effect).

The method of TWEPL was originally used to detect trap levels in GaP [3]. By noticing a saturating behavior of the BGE effect with increasing BGE density, we have improved the method to a quantitative one [4-6]. It allows us to choose a specific below-gap state by selecting the BGE energy and determine its density, carrier capture rates and spatial distribution quantitatively when proper BGE source is provided [6]. In continuation to our previous TWEPL characterization of a modulation-doped GaAs/AlGaAs QW [7], InGaN/GaN and GaN/AlGaN QW's [6, 8-11], we clarified an improved quality of plasma assisted (PA)-MBE grown GaN/AlGaN QW's [12-14] by increasing buffer-layer thickness and QW periods [15].

\footnotetext{
a e-mail: kamata@fms.saitama-u.ac.jp
} 


\section{Experimental}

An AlN-buffer layer $(250 \mathrm{~nm})$, and ten periods of $\mathrm{GaN}(3 \mathrm{~nm}) / \mathrm{Al}_{0.43} \mathrm{Ga}_{0.67} \mathrm{~N}(14 \mathrm{~nm}) \mathrm{QW}$ layers were grown in sequence on sapphire substrates by plasma assisted MBE [16]. Sample G65 was undoped, while G68 was Sidoped in both $\mathrm{GaN}$ well and $\mathrm{Al}_{0.43} \mathrm{Ga}_{0.67} \mathrm{~N}$ barrier layers. Electron concentrations obtained by Hall measurement were $6.4 \times 10^{18} \mathrm{~cm}^{-3}$ for G65 and $1.9 \times 10^{18} \mathrm{~cm}^{-3}$ for G68, respectively.

Aiming at higher emission efficiency, we next grew another F-series samples which have a GaN-nucleation layer $(20 \mathrm{~nm})$, an $\mathrm{Al}_{0.50} \mathrm{Ga}_{0.50} \mathrm{~N}$-buffer layer $(70 \mathrm{~nm})$ and 20 periods of $\mathrm{GaN}(4 \mathrm{~nm}) / \mathrm{Al}_{0.12} \mathrm{Ga}_{0.88} \mathrm{~N}(20 \mathrm{~nm}) \mathrm{QW}$ layers on sapphire substrates [15]. Sample F32 was undoped, while F37 was modulation-doped (i.e. Si atoms were introduced only inside the $\mathrm{Al}_{0.12} \mathrm{Ga}_{0.88} \mathrm{~N}$ barrier layers). Electron concentration of F37 was $9.5 \times 10^{18} \mathrm{~cm}^{-3}$, while that of F32 was about $4 \times 10^{18} \mathrm{~cm}^{-3}$.

In the TWEPL measurement, each sample was placed in a temperature-controlled cryostat and illuminated by both a continuous AGE light and a shuttered BGE light. Its band-edge PL intensity with and without the BGE, $\mathrm{I}_{\mathrm{AGE}+\mathrm{BGE}}$ and $\mathrm{I}_{\mathrm{AGE}}$, respectively, are measured to obtain the normalized PL intensity $\mathrm{I}_{\mathrm{AGE}+\mathrm{BGE}} / \mathrm{I}_{\mathrm{AGE}}$. Its deviation from unity corresponds to the presence of the below-gap state whose energy is matched with that of the BGE [6-11]. Lowering the AGE power improves the sensitivity, so the scheme of single-photon-counting was used to detect the band-edge PL [5]. In this experiment a Nd:YAG laser $(1.17 \mathrm{eV})$ was used as the BGE source, and a suitable photon energy as the AGE was selected from a $\mathrm{D}_{2}$ lamp by a monochromator. We consider that our sampling at BGE energy of $1.17 \mathrm{eV}$ is one of effective means for monitoring the distribution of below-gap states which affect the PL intensity up to room temperature [15].

\section{Effect of Si-doping}

Photoluminescence spectra of the undoped QW (G65) and the Si-doped one (G68) excited by the photon energy of $4.77 \mathrm{eV}$ at $77 \mathrm{~K}$ are shown in Figure 1. The latter exhibits higher PL intensity and narrower FWHM in comparison to those of the former. It is known that Si-doping yields a surfactant effect during growth $[1,2]$ and is effective for improving the crystal quality through stress relaxation and the reduction of imperfections. Lower electron concentration of G68 indicates that Si-doping actually reduces otherwise uncontrollable below-gap states.

We performed TWEPL measurement of G65 and G68 by an AGE energy of $4.88 \mathrm{eV}$. The temperature dependence of the BGE effect for samples G65 and G68 is shown in Figure 2. The normalized PL intensity of G65 decreased slightly below $50 \mathrm{~K}$, showing the value 0.93 at $15 \mathrm{~K}$. The value of $\mathrm{G} 68$ at $15 \mathrm{~K}$ is 0.98 and is close to unity up to $100 \mathrm{~K}$. Since the decrease in the normalized PL intensity at low temperature is attributed to the density of a pair of below-gap states described by our two-levels model [4-6], the result implies that the density of such pairs in G68 is

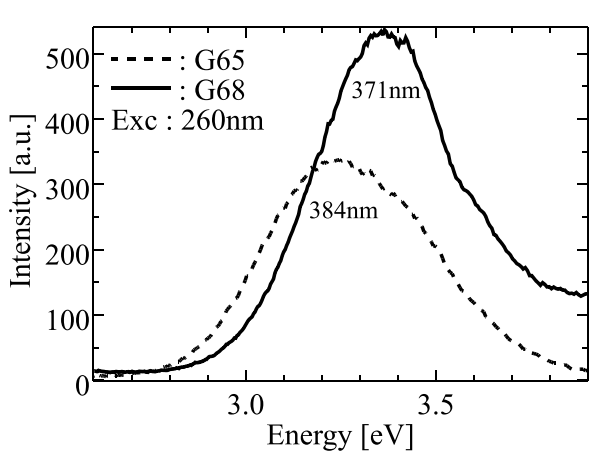

Fig. 1. PL spectra of samples G68 ( Si-doped QW) and G65 (undoped QW) at $77 \mathrm{~K}$.

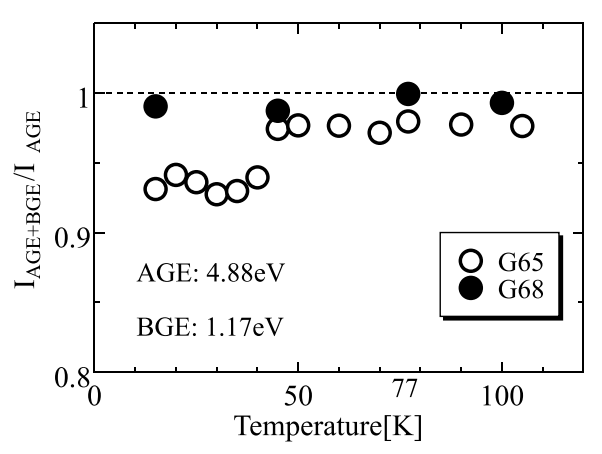

Fig. 2. The normalized PL intensity of G68 and G65 as a function of temperature.

lower than that in G65. The thermal emission of electrons from an upper state to the conduction band explains the temperature dependence [11].

\section{Improved PL properties by modulation-doping}

It was exemplified that the increase of hetero-interfaces reduces threading dislocations in both MOCVD- [9] and MBE-grown GaN/AlGaN QW's [12,15,17]. Combining with the previous result of efficiency improvement by modulation-doping [7], we studied F37 which has 20 periods of modulation-doped QW's in comparison with undoped equivalent F32.

Temperature dependence of their PL spectra excited by a photon energy of $4.12 \mathrm{eV}$ with an excitation density of $340 \mathrm{nW} / \mathrm{mm}^{2}$ is shown in Figure 3. The modulation-doped F37 showed stronger overall PL intensity, by a factor of 4 at $13 \mathrm{~K}$, which is not able to attribute to the difference in electron concentration. Its spectral shape contains a shoulder at about $3.36 \mathrm{eV}$ in low temperature region and this component becomes principal at the temperature above $100 \mathrm{~K}$. The undoped F32, on the other hand, shows a distinct sub-peak at $3.15 \mathrm{eV}$ even at $13 \mathrm{~K}$, and it becomes dominant above $40 \mathrm{~K}$. The lower-energy emission band indicates a distribution of below-gap states, some of which 


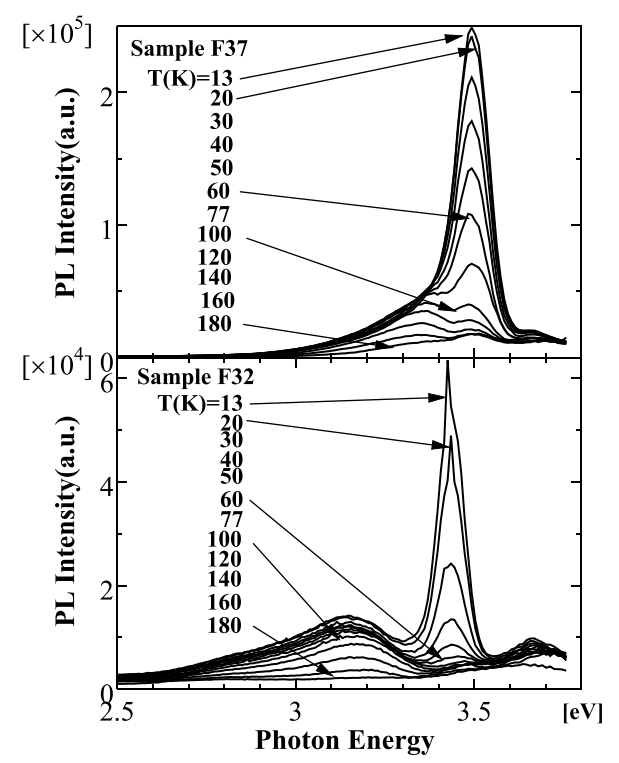

Fig. 3. PL spectra of F37 (upper) and F32 (lower), respectively, at the temperature range between $13 \mathrm{~K}$ and $180 \mathrm{~K}$.

act as NRR centers. Our TWEPL data of BGE energy dependence [15] suggests that the energy distribution of below-gap states in our MBE grown QW agrees well with those in a MOCVD grown QW [18].

In TWEPL measurement, we used a photon energy of $4.12 \mathrm{eV}$ with an excitation density of $5.5 \mathrm{nW} / \mathrm{mm}^{2}$ for AGE and a photon energy of $1.17 \mathrm{eV}$ with the density of $3.6 \mathrm{~mW} / \mathrm{mm}^{2}$ for BGE, respectively. Shown in Figure 4 is the resultant normalized PL intensity of F37 and F32 as a function of temperature. The PL intensity change of

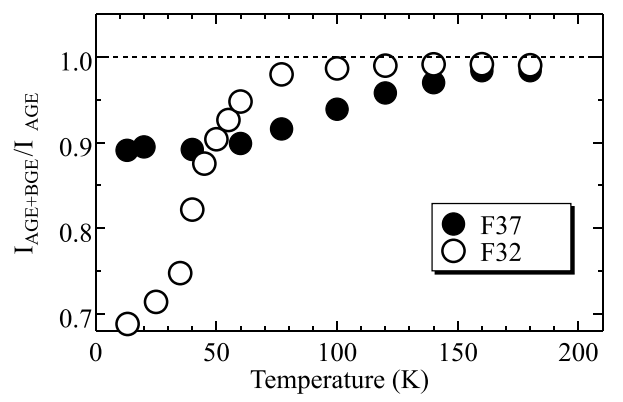

Fig. 4. The normalized PL intensity of F37 (Si modulationdoped QW) and F32 (undoped QW) as a function of temperature.

F37 due to the BGE at $15 \mathrm{~K}, 11 \%$ decrease, is less dominant than $32 \%$ decrease in that of F32, indicating lower concentration of below-gap states detected at the BGE energy of $1.17 \mathrm{eV}$ in the modulation-doped QW. This is consistent with the PL intensity as well as spectral shape shown in Figure 3. Corresponding to the composition of well and barrier layers, optimum profile of impurity concentration should be designed for minimizing below-gap states yet providing sufficient majority carriers in the well layer. We consider that wide-gap nature of GaN-based system is suitable for such optimisation.

\section{Conclusions}

We grew undoped and Si-doped GaN/AlGaN QW's by plasma assisted MBE and studied their below-gap states by the TWEPL measurement. By comparing the undoped sample, the Si-doped sample with $10 \mathrm{QW}$ periods showed lower density of below-gap states and higher internal quantum efficiency. The density of below-gap states in the modulation-doped sample with $20 \mathrm{QW}$ periods was also lower than that of the undoped one, which is consistent with the improved intensity and spectral shape of PL. These results are attributed to the stress relaxation and surfactant effect of Si doping during growth. Considering wide-gap nature, we conclude that modulation-doping is a promising way of improving crystal quality in GaN-based system.

The authors would like to thank Dr. K. Hoshino for valuable discussion, Mr. N. Gokan and Mr. F. Takahashi for supporting experiments. They also thank Japan Society for the Promotion of Science (Grant-in-Aid for Scientific Research (B)) and Shimadzu Science Foundation for their financial supports.

\section{References}

1. S. Nakamura, in Gallium Nitride I, Semicond. and Semimetals Vol. 50 (Academic Press, 1998); I. Akasaki, H. Amano, ibid.

2. G. Popovici, H. Morkoc, in GaN and Related Materials II, edited by S. J. Pearton (Gordon and Breach Sci. Publ., 2000)

3. H. G. Grimmeis, B. Monemar, Phys. Stat. Sol. A 19, 505 (1973)

4. E. Kanoh et al., J. Lumin. 63, 235 (1995)

5. N. Kamata et al., J. Lumin. 72-74, 797 (1997)

6. N. Kamata et al., Recent Res. Dev. Quantum Electron. 1, 123 (1999)

7. K. Hoshino et al., Physica E 7, 563 (2000)

8. J. M. Z. Ocampo et al., Phys. Stat. Sol. B 228, 433 (2001)

9. N. Kamata et al., Proc. 25th Int. Conf. Physics of Semiconductors, Springer Proc. in Phys. 87, 1521 (2001)

10. N. Kamata et al., Mater. Sci. Eng. B 91-92, 290 (2002)

11. N. Kamata et al., Inst. Phys. Conf. Ser. 170, 843 (2002)

12. J. Stemmer et al., J. Cryst. Growth 192, 471 (1998)

13. J. M. Z. Ocampo et al., Phys. Stat. Sol. A 183, 189 (2001)

14. F. Fedler et al., J. Cryst. Growth 241, 535 (2002)

15. H. Klausing et al., in Technical Digest of Int. Conf. on Nitride Semiconductors, Nara, 2003, edited by K. Onabe, p. 252

16. M. A. L. Johnson et al., J. Vac. Sci. Technol. B 14, 2349 (1996)

17. L. K. Li et al., J. Vac. Sci. Technol. B 18, 1472 (2000)

18. E. Calleja et al., Phys. Rev. B 55, 4689 (1997) 